\title{
Quinazoline-containing Hydrazydes of Dicarboxylic Acids and Products of Their Structural Modification: A Novel Class of Anti-inflammatory Agents
}

\author{
Nataliia Krasovska, ${ }^{1}$ Viktor Stavytskyi, ${ }^{1}$ Inna Nosulenko, ${ }^{2}$ \\ Oleksandr Karpenko, ${ }^{3}$ Oleksii Voskoboinik, ${ }^{1}$ and Serhii Kovalenko ${ }^{1, *}$
}

${ }^{1}$ Department of Organic and Bioorganic Chemistry, Zaporizhzhia State Medical University, 26 Maiakovski av., Zaporizhzhia, 69035, Ukraine

${ }^{2}$ Department of Pharmacognosy, Pharmacology and Botany, Zaporizhzhia State Medical University, 26 Maiakovski ave., Zaporizhzhia, 69035, Ukraine

${ }^{3}$ Enamine Ltd., Chervonotkatska Street 78, Kyiv 02094, Ukraine

*Corresponding author: E-mail: kovalenko.si@zsmu.zp.ua

Received: 10-09-2020

\begin{abstract}
The synthesis of hydrazides formed by quinazolin-4 $(3 H)$-ylidenehydrazine and dicarboxylic acids, as well as their further modification are described in the present manuscript. It was shown that above-mentioned hydrazides may be obtained via acylation of initial quinazolin- $4(3 H)$-ylidenehydrazine by corresponding acylhalides, cyclic anhydrides and imidazolides of dicarboxylic acids monoesters. Obtained hydrazides were converted into $[1,2,4]$ triazolo[1,5-c]quinazolines that were used as initial compounds for chemical modification aimed to the introduction of amide fragment to the molecule. The IR, ${ }^{1} \mathrm{H}$ NMR and chromato-mass spectral data of obtained compounds were studied and discussed. Obtained substances were studied for anti-inflammatory activity using carrageenan-induced paw inflammation model. Amides of $([1,2,4]$ triazolo[1,5-c] quinazoline-2-yl)alkyl carboxylic acids were detected as promising class of anti-inflammatory agents for further purposeful synthesis and profound study of anti-inflammatory activity.
\end{abstract}

Keywords: $[1,2,4]$ triazolo[1,5-c]quinazolines, quinazolines; anti-inflammatory activity

\section{Introduction}

The search for new biologically active compounds and the further development of drugs based on them is one of the most important tasks of medicinal and organic chemistry. It should be noted that elaboration of the new biologically active agents is a multistep process and choice of the research strategy and objects of investigation are quite important stages. Hydrazides formed by quinazolin-4(3H)-ylidenehydrazine are one of the promising objects for studies aimed to the development of novel pharmacologically active substances. Such high potential of above-mentioned compounds caused by the possibility of chemical modification aimed to the introduction of diverse pharmacophore fragments. ${ }^{1-7}$ Moreover, cyclisation of above-mentioned hydrazides yielded substituted triazolo $[c]$ quinazolines that show a wide range of biological activity including anticonvulsant, antitumor, hypoglycemic, antibacterial and other activities..$^{8-21}$ Despite the numerous publications devoted to the chemistry and biology of hydrazides formed by quinazolin-4(3H)-ylidenehydrazine, some features of their formation, reactivity, physicochemical and biological properties have been insufficiently studied. One of the promising directions of studies is the synthesis and further cyclization of hydrazides formed by quinazolin-4(3H)-ylidenehydrazine and dicarboxylic acids or their monoesters. These transformations would allow to combine heterocyclic fragments with quinazoline or $[1,2,4]$ triazolo[ $1,5-c]$ quinazoline heterocyclic fragment, what is reasonable in scope of elaboration of novel anti-inflammatory agents.

Therefore, the aim of the present study is to develop procedures for the synthesis of hydrazides formed by quinazolin-4 $(3 H)$-ylidenehydrazine and derivatives of di- 
carboxylic acids. Also the purpose was to study their cyclization, further modification of obtained tricyclic derivatives, as well as to study physicochemical properties and anti-inflammatory activity of obtained products.

\section{Experimental Section}

Melting points were determined in open capillary tubes in a «Stuart SMP30» apparatus and are uncorrected. The elemental analyses $(\mathrm{C}, \mathrm{H}, \mathrm{N})$ were performed using the «ELEMENTAR vario EL cube» analyzer. IR spectra (4000-600 $\mathrm{cm}^{-1}$ ) were recorded on a Bruker ALPHA FTIR spectrometer using a module ATR eco ZnSe. ${ }^{1} \mathrm{H}$ NMR spectra $(400 \mathrm{MHz})$ were recorded on a Varian-Mercury 400 (Varian Inc., Palo Alto, CA, USA) spectrometer with TMS as internal standard in DMSO- $d_{6}$ solution. LC-MS were recorded using chromatography/mass spectrometric system which consists of high performance liquid chromatograph "Agilent 1100 Series» (Agilent, Palo Alto, CA, USA) equipped with diode-matrix and mass-selective detector "Agilent LC/MSD SL» (atmospheric pressure chemical ionization - APCI). The purity of all obtained compounds was checked by ${ }^{1} \mathrm{H}$ NMR and LC-MS.

Compound 1a was synthesized according to the reported procedures. ${ }^{1-3}$ Other starting materials and solvents were obtained from commercially available sources and were used without additional purification.

\subsection{General Method for the Synthesis of 2-(4(3H)-Quinazolinylidene)hydrazides of Dicarboxylic Acids and Their Monoesters (2a-f)}

Method A. $1.11 \mathrm{~g}(11 \mathrm{mmol})$ of triethylamine was added to the suspension of $1.6 \mathrm{~g}(10 \mathrm{mmol})$ of 4-hydrazinoquinazoline (1a) in $10 \mathrm{~mL}$ of dioxane. The formed mixture was cooled to $0-5{ }^{\circ} \mathrm{C}$ and $11 \mathrm{mmol}$ of ethyl 2-chloro-2-oxoacetate or ethyl 3-chloro-3-oxopropanoate was added under stirring. The formed mixture was stirred for $1.5 \mathrm{~h}$ at $0-5{ }^{\circ} \mathrm{C}$, then poured in saturated solution of sodium acetate. The formed mixture was filtered off and dried. For additional purification compounds $\mathbf{2 a}$ and $\mathbf{2 b}$ may be crystallized from methanol.

Method B. $1.78 \mathrm{~g}(11 \mathrm{mmol})$ of $N, N^{\prime}$-carbonyldiimidazole (CDI) was added to the solution of corresponding monoethyl ester of dicarboxylic acid in $20 \mathrm{~mL}$ of anhydrous dioxane. The formed mixture was heated at $80{ }^{\circ} \mathrm{C}$ for $1 \mathrm{~h}$ (until the carbon dioxide was completely released). Then 1.6 $\mathrm{g}(10 \mathrm{mmol})$ of 4-hydrazinoquinazoline (1a) was added and stirred for 1.5-3 $\mathrm{h}$. The formed mixture was cooled and poured into water and acidified to $\mathrm{pH} 5-6$. The formed mixture was filtered off and dried. For additional purification compounds $\mathbf{2} \mathbf{a}$ and $\mathbf{2 b}$ may be crystallized from methanol.

Compounds $\mathbf{2 a}$ and $\mathbf{2 b}$ that were synthesized by methods A and B have identical physicochemical properties.
Method C. $11 \mathrm{mmol}$ of corresponding anhydride of dicarboxylic acid under stirring was added to the suspension of $1.6 \mathrm{~g}(10 \mathrm{mmol})$ of 4 -hydrazinoquinazoline (1a) in 10 $\mathrm{mL}$ of dioxane. Formed mixture was stirred at ambient temperature for $24 \mathrm{~h}$ or at $80^{\circ} \mathrm{C}$ for $1-1.5 \mathrm{~h}$. Then, reaction mixture was cooled, and the formed mixture was filtered off, washed by ethanol and dried. For additional purification obtained compounds may be crystallized from methanol.

Ethyl 2-oxo-2-(2-(quinazolin-4(3H)-ylidene)hydrazineyl)acetate (2a). Yield: $1.83 \mathrm{~g}(70 \%)(\operatorname{method} \mathrm{A}), 2.25 \mathrm{~g}$ (86\%) (method B). Mp 199-202 ${ }^{\circ} \mathrm{C}$; IR $3007\left(v_{\mathrm{NH}}\right), 1741$ $\left(v_{\mathrm{CO}}\right), 1689\left(v_{\mathrm{CO}}\right), 1616\left(\delta_{\mathrm{NH}}\right), 1546,1444,1110\left(v_{\mathrm{COC}}\right)$, $760,688 \mathrm{~cm}^{-1} .{ }^{1} \mathrm{H}$ NMR $\left(400 \mathrm{MHz}, \mathrm{DMSO}-d_{6}\right) \delta 1.36(\mathrm{t}, J$ $\left.=7.1 \mathrm{~Hz}, 3 \mathrm{H},-\mathrm{CH}_{3}\right), 4.28\left(\mathrm{q}, J=7.1 \mathrm{~Hz}, 2 \mathrm{H},-\mathrm{CH}_{2}-\right), 7.17$ $(\mathrm{d}, J=7.8 \mathrm{~Hz}, 1 \mathrm{H}, \mathrm{H}-8), 7.26(\mathrm{t}, J=7.9 \mathrm{~Hz}, 1 \mathrm{H}, \mathrm{H}-6), 7.42$ (t, $J=7.9 \mathrm{~Hz}, 1 \mathrm{H}, \mathrm{H}-7), 7.91(\mathrm{~s}, 1 \mathrm{H}, \mathrm{H}-2), 8.02(\mathrm{~d}, J=7.8$ $\mathrm{Hz}, 1 \mathrm{H}, \mathrm{H}-5$ ), 11.11 (br. s, $1 \mathrm{H},-\mathrm{NH}-$ ), 11.79 (br. s, $1 \mathrm{H}$, $-N H)$. LC-MS $m / z=261[\mathrm{M}+1]$; Anal. Calcd. for $\mathrm{C}_{12} \mathrm{H}_{12} \mathrm{~N}_{4} \mathrm{O}_{3}$ : C, 55.38; H, 4.65; N, 21.53; Found: C, 55.46; $\mathrm{H}, 4.71 ; \mathrm{N}, 21.58$.

Ethyl 3-oxo-3-(2-(quinazolin-4(3H)-ylidene)hydrazineyl)propanoate (2b). Yield: $1.93 \mathrm{~g}(70 \%)$ (method A), 2.42 g (88.3\%) (method B). Mp $165-167{ }^{\circ} \mathrm{C}$; IR $3250\left(v_{\mathrm{NH}}\right)$, $3198\left(v_{\mathrm{NH}}\right), 2986\left(v_{\mathrm{CH} 2}\right), 1723\left(v_{\mathrm{CO}}\right), 1656\left(v_{\mathrm{CO}}\right), 1519$ $\left(\delta_{\mathrm{NH}}\right), 1435,1309,1158\left(v_{\mathrm{COC}}\right), 1023,987,759,640 \mathrm{~cm}^{-1}$. ${ }^{1} \mathrm{H}$ NMR $\left(400 \mathrm{MHz}\right.$, DMSO- $\left.d_{6}\right) \delta 1.24(\mathrm{t}, J=7.1 \mathrm{~Hz}, 3 \mathrm{H}$, $\left.\left.-\mathrm{CH}_{2} \mathrm{C}_{3}\right), 3.46\left(\mathrm{~s}, 2 \mathrm{H},-\mathrm{C}_{2}\right)^{-}\right), 4.16(\mathrm{q}, J=7.1 \mathrm{~Hz}, 2 \mathrm{H}$, $\left.-\mathrm{CH}_{2} \mathrm{CH}_{3}\right), 7.17(\mathrm{~d}, J=7.8 \mathrm{~Hz}, 1 \mathrm{H}, \mathrm{H}-8), 7.26(\mathrm{t}, J=7.9 \mathrm{~Hz}$, $1 \mathrm{H}, \mathrm{H}-6), 7.42(\mathrm{t}, J=7.9 \mathrm{~Hz}, 1 \mathrm{H}, \mathrm{H}-7), 7.91(\mathrm{~s}, 1 \mathrm{H}, \mathrm{H}-2)$, 8.02 (d, $J=7.8 \mathrm{~Hz}, 1 \mathrm{H}, \mathrm{H}-5), 10.66$ (br. s, $1 \mathrm{H},-\mathrm{NH}$-), 11.10 (br. s, $1 \mathrm{H},-\mathrm{NH}$-). LC-MS $m / z=275$ [M+1]; Anal. Calcd. for $\mathrm{C}_{13} \mathrm{H}_{14} \mathrm{~N}_{4} \mathrm{O}_{3}$ : C, 56.93; H, 5.15; N, 20.43; Found: C, 57.02; H, 5.19; N, 20.48.

4-Oxo-4-(2-(quinazolin-4(3H)-ylidene)hydrazineyl)butanoic acid (2c). Yield: $2.43 \mathrm{~g}(93 \%)$ (method C). Mp 177-179 ${ }^{\circ} \mathrm{C}$; IR $3270\left(v_{\mathrm{OH}}\right), 3258\left(v_{\mathrm{NH}}\right), 1703\left(v_{\mathrm{CO}}\right), 1602$ $\left(v_{\mathrm{CO}}\right), 1555\left(\delta_{\mathrm{NH}}\right), 1527,1442,1212,929\left(\delta_{\mathrm{OH}}\right), 740,687$ $\mathrm{cm}^{-1} .{ }^{1} \mathrm{H}$ NMR $\left(400 \mathrm{MHz}\right.$, DMSO- $\left.d_{6}\right) \delta 2.91(\mathrm{~m}, 2 \mathrm{H}$, $\left.-\mathrm{CH}_{2} \mathrm{C}_{2}\right), 3.58\left(\mathrm{~m}, 2 \mathrm{H},-\underline{\mathrm{C}}_{2} \mathrm{CH}_{2}\right), 7.10(\mathrm{~d}, J=7.7 \mathrm{~Hz}, 1 \mathrm{H}$, $\mathrm{H}-8), 7.59$ and $7.20(2 \times \mathrm{t}, J=7.6 \mathrm{~Hz}, 1 \mathrm{H}, \mathrm{H}-6), 7.52$ and 7.36 $(2 \times \mathrm{t}, J=7.6 \mathrm{~Hz}, 1 \mathrm{H}, \mathrm{H}-7), 8.25$ and $7.74(2 \times \mathrm{s}, 1 \mathrm{H}, \mathrm{H}-2), 8.04$ and $7.88(2 \times \mathrm{d}, J=7.5 \mathrm{~Hz}, 1 \mathrm{H}, \mathrm{H}-5), 10.01$ and $9.52(2 \times \mathrm{s}, 1 \mathrm{H}$, -NH-), 11.37 and $10.88(2 \times \mathrm{s}, 1 \mathrm{H},-\mathrm{NH}-)$. LC-MS $m / z=261$ [M+1]; Anal. Calcd. for $\mathrm{C}_{12} \mathrm{H}_{12} \mathrm{~N}_{4} \mathrm{O}_{3}$ : C, 55.38; $\mathrm{H}, 4.65 ; \mathrm{N}$, 21.53; O, 18.44; Found: C, 55.46; H, 4.69; N, 21.66.

5-Oxo-5-(2-(quinazolin-4(3H)-ylidene)hydrazineyl) pentanoic acid (2d). Yield: $2.73 \mathrm{~g}$ (99\%) (method C). Mp 133-135 ${ }^{\circ} \mathrm{C}$; IR $3356\left(v_{\mathrm{OH}}\right), 3204\left(v_{\mathrm{NH}}\right), 2935\left(v_{\mathrm{CH} 2}\right), 1705$ $\left(v_{\mathrm{CO}}\right), 1635\left(v_{\mathrm{CO}}\right), 1566\left(\delta_{\mathrm{NH}}\right), 1537,1369,1257,792,763$, $684 \mathrm{~cm}^{-1} .{ }^{1} \mathrm{H}$ NMR (400 MHz, DMSO- $\left.d_{6}\right) \delta 1.87(\mathrm{~m}, 2 \mathrm{H}$, $-\mathrm{CH}_{2} \mathrm{C}_{2} \mathrm{CH}_{2}$ ) $), 2.30\left(\mathrm{~m}, 2 \mathrm{H},-\mathrm{CH}_{2} \mathrm{CH}_{2} \mathrm{C}_{2}\right), 2.68(\mathrm{~m}, 2 \mathrm{H}$, $\left.-\mathrm{C}_{2} \mathrm{CH}_{2} \mathrm{CH}_{2-}\right), 7.08(\mathrm{~d}, J=7.7 \mathrm{~Hz}, 1 \mathrm{H}, \mathrm{H}-8), 7.50$ and 
$7.19(2 \times \mathrm{t}, J=7.8 \mathrm{~Hz}, 1 \mathrm{H}, \mathrm{H}-6), 7.52$ and $7.36(2 \times \mathrm{t}, J=7.7$ $\mathrm{Hz}, 1 \mathrm{H}, \mathrm{H}-7), 7.73$ (s, $1 \mathrm{H}, \mathrm{H}-2), 8.11$ and $7.87(2 \times \mathrm{d}, J=7.6$ $\mathrm{Hz}, 1 \mathrm{H}, \mathrm{H}-5), 9.93$ and $9.48(2 \times \mathrm{s}, 1 \mathrm{H},-\mathrm{NH}-), 11.80$ and $11.35(2 \times s, 1 \mathrm{H},-\mathrm{NH}-)$. LC-MS $m / z=275[\mathrm{M}+1]$; Anal. Calcd. for $\mathrm{C}_{13} \mathrm{H}_{14} \mathrm{~N}_{4} \mathrm{O}_{3}$ : C, 56.93; H, 5.15; N, 20.43; Found: C, 56.99; H, 5.21; N, 20.50.

3-Methyl-5-oxo-5-(2-(quinazolin-4(3H)-ylidene)hydrazineyl)pentanoic acid (2e). Yield: $2.86 \mathrm{~g}$ (99\%) (method C). Mp 170-173 ${ }^{\circ} \mathrm{C}$; IR $3724\left(v_{\mathrm{OH}}\right), 3256\left(v_{\mathrm{NH}}\right), 2928$ $\left(v_{\mathrm{CH} 2}\right), 1720\left(v_{\mathrm{CO}}\right), 1600\left(v_{\mathrm{CO}}\right), 1530\left(\delta_{\mathrm{NH}}\right), 1371,871,760$, $688 \mathrm{~cm}^{-1} .{ }^{1} \mathrm{H}$ NMR (400 MHz, DMSO- $\left.d_{6}\right) \delta 1.03-1.01(\mathrm{~m}$, $\left.\left.3 \mathrm{H}, \quad-\mathrm{CH}_{2} \mathrm{CH}\left(\mathrm{CH}_{3}\right) \mathrm{CH}_{2^{-}}\right), \mathrm{CH}_{3}\right), \quad 2.66-2.07(\mathrm{~m}, 5 \mathrm{H}$, $\left.-\mathrm{C} \underline{H}_{2} \mathrm{C} \underline{H}\left(\mathrm{CH}_{3}\right) \underline{\mathrm{C}}_{2^{-}}\right), 7.09(\mathrm{~d}, J=7.5 \mathrm{~Hz}, 1 \mathrm{H}, \mathrm{H}-8), 7.19(\mathrm{t}$, $J=6.6 \mathrm{~Hz}, 1 \mathrm{H}, \mathrm{H}-6), 7.45-7.25$ (m, 1H, H-7), 7.73 (s, 1H, $\mathrm{H}-2), 7.87(\mathrm{~d}, J=7.5 \mathrm{~Hz}, 1 \mathrm{H}, \mathrm{H}-5), 9.93$ and $9.50(2 \times \mathrm{s}, 1 \mathrm{H}$, $-N H-), 11.80$ and 11.35 (2×s, $1 \mathrm{H},-N H-)$. LC-MS $m / z=289$ $[\mathrm{M}+1]$; Anal. Calcd. for $\mathrm{C}_{14} \mathrm{H}_{16} \mathrm{~N}_{4} \mathrm{O}_{3}$ : C, 58.32; $\mathrm{H}, 5.59 ; \mathrm{N}$, 19.43; Found: C, 58.37; H, 5.63; N, 19.49.

2-(1-(2-Oxo-2-(2-(quinazolin-4(3H)-ylidene)hydrazineyl)ethyl)cyclopentyl)acetic acid (2f). Yield: $2.63 \mathrm{~g}(80 \%)$ (method C). Mp 189-191 ${ }^{\circ} \mathrm{C}$; IR $3694\left(v_{\mathrm{OH}}\right), 3256\left(v_{\mathrm{NH}}\right)$, $2988\left(v_{\mathrm{CH} 2}\right), 1703\left(v_{\mathrm{CO}}\right), 1692\left(v_{\mathrm{CO}}\right), 1580\left(\delta_{\mathrm{NH}}\right), 1524,1329$, $938\left(\delta_{\mathrm{OH}}\right), 796,668 \mathrm{~cm}^{-1} .{ }^{1} \mathrm{H}$ NMR $\left(400 \mathrm{MHz}, \mathrm{DMSO}-d_{6}\right) \delta$ 1.88-1.38 (m, 8H, $-\mathrm{CH}_{2}$ (cyclopenthyl) $\left.\mathrm{CH}_{2^{-}}\right), 2.90$ and 2.45 $\left(2 \times \mathrm{m}, 4 \mathrm{H},-\underline{C}_{2}(\right.$ cyclopenthyl $\left.) \underline{\mathrm{C}}_{2^{-}}\right), 7.10(\mathrm{~d}, J=7.0 \mathrm{~Hz}$, $1 \mathrm{H}, \mathrm{H}-8), 7.24-7.14$ (m, 1H, H-6), 7.45-7.29 (m, 1H, H-7), 7.75 (s, 1H, H-2), 7.85 (d, $J=7.4 \mathrm{~Hz}, 1 \mathrm{H}, \mathrm{H}-5), 10.01$ and $9.59(2 \times \mathrm{s}, 1 \mathrm{H},-\mathrm{NH}-), 11.90$ and $11.40(2 \times \mathrm{s}, 1 \mathrm{H},-\mathrm{NH}-)$. LC-MS $m / z=329[\mathrm{M}+1]$; Anal. Calcd. for $\mathrm{C}_{17} \mathrm{H}_{20} \mathrm{~N}_{4} \mathrm{O}_{3}$ : 62.18; H, 6.14; N, 17.06; Found: C, 62.23; H, 6.19; N, 17.12.

\section{2. General Method for the Synthesis of $([1,2,4]$ Triazolo $[1,5-c]$ quinazolin-2-yl) carboxylic Acids and Their Esters (3a-b)}

Method A. The solution of $5 \mathrm{mmol}$ of corresponding quinazoline-containing hydrazide of dicarboxylic acid (2c-f) or ester (2a, 2b) in $20 \mathrm{~mL}$ of acetic acid was refluxed for 3-4 h with removing of formed water. After completing of reaction, the solvent was evaporated under vacuum. 30 $\mathrm{mL}$ of methanol was added to the residue and mixture was shaken. The formed precipitate was filtered, washed by 10 $\mathrm{mL}$ of ether and dried. For additional purification, compounds $\mathbf{3 a}-\mathbf{f}$ may be crystallized from ethanol (3a, $\mathbf{3 b}$ ) or dioxane (3c-f).

Method B. $5.5 \mathrm{mmol}$ of sodium acetate was added to the suspension of $0.8 \mathrm{~g}$ ( $5 \mathrm{mmol})$ of 4-hydrazinoquinazoline (1a) in $10 \mathrm{~mL}$ of glacial acetic acid. The formed mixture was cooled to $0-5{ }^{\circ} \mathrm{C}$ and $5.5 \mathrm{mmol}$ of ethyl 2-chloro-2-oxoacetate or ethyl 3-chloro-3-oxopropanoate was added dropwise under stirring. The formed mixture was stirred for $1.5 \mathrm{~h}$ and then refluxed for $3 \mathrm{~h}$. The formed precipitate of sodium chloride was filtered off, the solvent was evaporated under vacuum, $10 \mathrm{~mL}$ of methanol was added and formed mixture was shaken. The formed precipitate was filtered, washed by $10 \mathrm{~mL}$ of ether and dried. For additional purification compounds $\mathbf{3 a}-\mathbf{f}$ may be crystallized from ethanol.

Method C. $5.5 \mathrm{mmol}$ of corresponding dicarboxylic acid anhydride was added to the solution of $0.8 \mathrm{~g}(5 \mathrm{mmol})$ 4-hydrazinoquinazoline (1a) in $20 \mathrm{~mL}$ of glacial acetic acid. The formed mixture was refluxed for 3-4 $\mathrm{h}$ with water removal. After completing of the reaction, the solvent was evaporated under vacuum, $10 \mathrm{~mL}$ of methanol was added and formed mixture was shaken. The formed precipitate was filtered off, washed by diethyl ether and dried. Compounds 3a-f may be additionally purified by crystallization from dioxane.

Compounds $\mathbf{3 a}, \mathbf{3} \mathbf{b}$ that were synthesized by methods A and B have identical physicochemical properties.

Ethyl $[1,2,4]$ triazolo[1,5-c] quinazoline-2-carboxylate (3a). Yield: $1.03 \mathrm{~g}$ (85\%) (method A), $0.87 \mathrm{~g}$ (72\%) (method B). Mp 172-175 ${ }^{\circ} \mathrm{C}$; IR $2920\left(v_{\mathrm{CH} 2}\right), 2851\left(v_{\mathrm{CH}}\right), 1730$ $\left(v_{\mathrm{CO}}\right), 1625,1517,1458,1363,1201$ ( $\left.v_{\mathrm{COC}}\right), 1019,862,780$, $708,654 \mathrm{~cm}^{-1} .{ }^{1} \mathrm{H}$ NMR (400 MHz, DMSO- $\left.d_{6}\right) \delta 1.44(\mathrm{t}, J$ $\left.=7.2 \mathrm{~Hz}, 3 \mathrm{H}, \underline{\mathrm{C}}_{3}\right), 4.46\left(\mathrm{q}, J=7.1 \mathrm{~Hz}, 2 \mathrm{H}, \mathrm{C}_{2}\right), 7.83(\mathrm{t}, J$ $=7.7 \mathrm{~Hz}, 1 \mathrm{H}, \mathrm{H}-9), 7.92(\mathrm{t}, J=7.7 \mathrm{~Hz}, 1 \mathrm{H}, \mathrm{H}-8), 8.05(\mathrm{~d}, J$ $=7.7 \mathrm{~Hz}, 1 \mathrm{H}, \mathrm{H}-7), 8.54(\mathrm{~d}, J=7.7 \mathrm{~Hz}, 1 \mathrm{H}, \mathrm{H}-10), 9.52(\mathrm{~s}$, $1 \mathrm{H}, \mathrm{H}-5)$. LC-MS $\mathrm{m} / z=243$ [M+1]; Anal. Calcd. for $\mathrm{C}_{12} \mathrm{H}_{10} \mathrm{~N}_{4} \mathrm{O}_{2}$ : C, 59.50; H, 4.16; N, 23.13; Found: C, 59.58; $\mathrm{H}, 4.21 ; \mathrm{N}, 23.19$.

Ethyl 2-([1,2,4]triazolo[1,5-c]quinazolin-2-yl)acetate (3b). Yield: $1.02 \mathrm{~g} \mathrm{(79 \% )} \mathrm{(method} \mathrm{A),} 0.73 \mathrm{~g}$ (57\%) (method B). Mp 125-127 ${ }^{\circ} \mathrm{C}$; IR $2944\left(v_{\mathrm{CH} 2}\right), 1722\left(v_{\mathrm{CO}}\right), 1621$, $1524,1370,1219$ ( $\left.v_{\text {COC }}\right), 1026,897,774,710,668 \mathrm{~cm}^{-1} .{ }^{1} \mathrm{H}$ NMR (400 MHz, DMSO- $\left.d_{6}\right) \delta 1.28(\mathrm{t}, J=7.1 \mathrm{~Hz}, 3 \mathrm{H}$, $\left.-\mathrm{CH}_{3}\right), 3.96\left(\mathrm{~s}, 2 \mathrm{H},-\underline{\mathrm{CH}}_{2^{-}}\right), 4.17(\mathrm{q}, J=7.1 \mathrm{~Hz}, 2 \mathrm{H}$, $\left.-\mathrm{CH}_{2} \mathrm{CH}_{3}\right), 7.77$ (t, $\left.J=7.5 \mathrm{~Hz}, 1 \mathrm{H}, \mathrm{H}-9\right), 7.87$ (t, $J=7.6 \mathrm{~Hz}$, $1 \mathrm{H}, \mathrm{H}-8), 8.02$ (d, J=8.2 Hz, 1H, H-7), 8.44 (d, J= $7.8 \mathrm{~Hz}$, $1 \mathrm{H}, \mathrm{H}-10$ ), 9.40 (s, $1 \mathrm{H}, \mathrm{H}-5)$. LC-MS $m / z=257[\mathrm{M}+1]$; Anal. Calcd. for $\mathrm{C}_{13} \mathrm{H}_{12} \mathrm{~N}_{4} \mathrm{O}_{2}$ : C, 60.93; $\mathrm{H}, 4.72 ; \mathrm{N}, 21.86$; Found: C, 61.02; H, 4.80; N, 21.94.

3-([1,2,4]Triazolo[1,5-c]quinazolin-2-yl)propanoic acid (3c). Yield: $1.20 \mathrm{~g}(99 \%)$ (method C). Mp 200-203 ${ }^{\circ} \mathrm{C}$; IR $2900\left(v_{\mathrm{CH} 2}\right), 1723\left(v_{\mathrm{CO}}\right), 1625,1502,1362,1338,1259$, $907\left(\delta_{\mathrm{OH}}\right), 782,710,668 \mathrm{~cm}^{-1} .{ }^{1} \mathrm{H}$ NMR $(400 \mathrm{MHz}$, DMSO- $\left.d_{6}\right) \delta 2.82\left(\mathrm{t}, J=7.3 \mathrm{~Hz}, 2 \mathrm{H},-\mathrm{CH}_{2} \mathrm{CH}_{2} \mathrm{COOH}\right)$, $3.18\left(\mathrm{t}, J=7.2 \mathrm{~Hz}, 2 \mathrm{H},-\mathrm{CH}_{2} \mathrm{CH}_{2} \mathrm{COOH}\right), 7.71(\mathrm{t}, J=7.6$ $\mathrm{Hz}, 1 \mathrm{H}, \mathrm{H}-9), 7.82$ (t, $J=7.6 \mathrm{~Hz}, 1 \mathrm{H}, \mathrm{H}-8), 7.98$ (d, $J=7.7$ $\mathrm{Hz}, 1 \mathrm{H}, \mathrm{H}-7), 8.42$ (d, J = 7.7 Hz, 1H, H-10), 9.26 (s, 1H, $\mathrm{H}-5$ ), 11.90 (br. s, $1 \mathrm{H},-\mathrm{COOH}$ ). LC-MS $m / z=243$ [M+1]; Anal. Calcd. for $\mathrm{C}_{12} \mathrm{H}_{10} \mathrm{~N}_{4} \mathrm{O}_{2}$ : C, 59.50; H, 4.16; N, 23.13; Found: C, 59.56; H, 4.20; N, 23.21.

4-([1,2,4]Triazolo[1,5-c]quinazolin-2-yl)butanoic acid (3d). Yield: $1.16 \mathrm{~g}$ (91\%) (method C). Mp 184-186 ${ }^{\circ} \mathrm{C}$; IR $2928\left(v_{\mathrm{CH} 2}\right), 1714\left(v_{\mathrm{CO}}\right), 1625,1521,1404,1366,1329$, 
1241, 1181, $909\left(\delta_{\mathrm{OH}}\right), 792,756,711,669 \mathrm{~cm}^{-1} .{ }^{1} \mathrm{H}$ NMR $\left(400 \mathrm{MHz}, \mathrm{DMSO}-d_{6}\right) \delta 2.10\left(\mathrm{~m}, 2 \mathrm{H},-\mathrm{CH}_{2} \mathrm{CH}_{2} \mathrm{CH}_{2} \mathrm{COOH}\right)$, $2.41\left(\mathrm{t}, J=7.2 \mathrm{~Hz}, 2 \mathrm{H},-\mathrm{CH}_{2}-\left(\mathrm{CH}_{2}\right)_{2} \mathrm{COOH}\right), 3.00(\mathrm{t}, J=$ $\left.7.2 \mathrm{~Hz}, 2 \mathrm{H},-\left(\mathrm{CH}_{2}\right)_{2} \mathrm{CH}_{2} \mathrm{COOH}\right), 7.73(\mathrm{t}, J=7.7 \mathrm{~Hz}, 1 \mathrm{H}$, H-9), $7.82(\mathrm{t}, J=7.7 \mathrm{~Hz}, 1 \mathrm{H}, \mathrm{H}-8), 8.00(\mathrm{~d}, J=7.7 \mathrm{~Hz}, 1 \mathrm{H}$, $\mathrm{H}-7), 8.44$ (d, $J=7.7 \mathrm{~Hz}, 1 \mathrm{H}, \mathrm{H}-10), 9.27$ (s, 1H, H-5), 11.82 (br. s, $1 \mathrm{H},-\mathrm{COOH}$ ). LC-MS $m / z=257$ [M+1]; Anal. Calcd. for $\mathrm{C}_{13} \mathrm{H}_{12} \mathrm{~N}_{4} \mathrm{O}_{2}$ : C, 60.93; $\mathrm{H}, 4.72 ; \mathrm{N}, 21.86$; Found: C, 60.99; H, 4.78; N, 21.94.

4-([1,2,4]Triazolo[1,5-c] quinazolin-2-yl)-3-methylbutanoic acid (3e). Yield: $0.70 \mathrm{~g}(52 \%)$ (method C). Mp $168-170^{\circ} \mathrm{C}$; IR $2958\left(v_{\mathrm{CH} 2}\right), 1714\left(v_{\mathrm{CO}}\right), 1628,1528,1470$, 1370, 1316, 1258, $911\left(\delta_{\mathrm{OH}}\right), 771,704,653 \mathrm{~cm}^{-1} .{ }^{1} \mathrm{H}$ NMR $\left(400 \mathrm{MHz}, \mathrm{DMSO}-d_{6}\right) \delta 1.05(\mathrm{~d}, J=6.2 \mathrm{~Hz}, 3 \mathrm{H}$, $\left.-\mathrm{CH}_{2} \mathrm{CH}\left(\mathrm{CH}_{3}\right) \mathrm{CH}_{2}-\right), 2.17\left(\mathrm{dd}, J^{2}=15.5 \mathrm{~Hz}, J^{3}=8.1 \mathrm{~Hz}\right.$, $\left.1 \mathrm{H},-\mathrm{CH}_{2} \mathrm{CH}\left(\mathrm{CH}_{3}\right) \mathrm{C}_{2^{-}}\right), 2.41\left(\mathrm{dd}, J^{2}=15.5 \mathrm{~Hz}, J^{3}=5.0\right.$ $\left.\mathrm{Hz}, \quad 1 \mathrm{H}, \quad-\mathrm{CH}_{2} \mathrm{CH}\left(\mathrm{CH}_{3}\right) \mathrm{CH}_{2}\right)^{-}, \quad 2.66-2.52 \quad(\mathrm{~m}, \quad 1 \mathrm{H}$, $\left.-\mathrm{CH}_{2} \mathrm{C} \underline{H}\left(\mathrm{CH}_{3}\right) \mathrm{CH}_{2}-\right), 2.83\left(\mathrm{dd}, J^{2}=14.0 \mathrm{~Hz}, J^{3}=7.5 \mathrm{~Hz}\right.$, $\left.1 \mathrm{H},-\mathrm{CH}_{2} \mathrm{CH}\left(\mathrm{CH}_{3}\right) \mathrm{CH}_{2}-\right), 2.96\left(\mathrm{dd}, J^{2}=14.0 \mathrm{~Hz}, J^{3}=6.0\right.$ $\left.\mathrm{Hz}, 1 \mathrm{H},-\mathrm{C}_{2} \mathrm{CH}\left(\mathrm{CH}_{3}\right) \mathrm{CH}_{2}-\right), 7.76(\mathrm{t}, J=7.5 \mathrm{~Hz}, 1 \mathrm{H}, \mathrm{H}-9)$, $7.86(\mathrm{t}, J=7.6 \mathrm{~Hz}, 1 \mathrm{H}, \mathrm{H}-8), 8.01(\mathrm{~d}, J=8.1 \mathrm{~Hz}, 1 \mathrm{H}, \mathrm{H}-7)$, 8.44 (d, $J=7.9 \mathrm{~Hz}, 1 \mathrm{H}, \mathrm{H}-10), 9.37$ (s, $1 \mathrm{H}, \mathrm{H}-5), 11.83$ (s, $1 \mathrm{H},-\mathrm{COOH}$ ). LC-MS $m / z=271$ [M+1]; Anal. Calcd. for $\mathrm{C}_{14} \mathrm{H}_{14} \mathrm{~N}_{4} \mathrm{O}_{2}$ : C, 62.21; H, 5.22; N, 20.73; Found: C, 62.29; $\mathrm{H}, 5.31 ; \mathrm{N}, 20.81$.

2-(1-([1,2,4]Triazolo[1,5-c] quinazolin-2-ylmethyl)cyclopentyl)acetic acid (3f). Yield: $0.79 \mathrm{~g}(51 \%)$ (method C). Mp $147-149{ }^{\circ} \mathrm{C}$; IR $2952\left(v_{\mathrm{CH} 2}\right), 2310,1706\left(v_{\mathrm{CO}}\right)$, $1620,1553,1515,1486,1353,1313,1237,931\left(\delta_{\mathrm{OH}}\right), 899$, 774, 728, $698 \mathrm{~cm}^{-1} .{ }^{1} \mathrm{H}$ NMR $\left(400 \mathrm{MHz}\right.$, DMSO- $\left.d_{6}\right) \delta$ 1.86-1.42 (m, $8 \mathrm{H},-\mathrm{CH}_{2}$ (cyclopenthyl) $\left.\mathrm{CH}_{2}-\right), 2.47-2.31(\mathrm{~m}$, $2 \mathrm{H}, \quad-\mathrm{CH}_{2}$ (cyclopenthyl) $\left.\mathrm{CH}_{2^{-}}\right), \quad 3.17-3.05 \quad(\mathrm{~m}, \quad 2 \mathrm{H}$, $-\mathrm{CH}_{2}($ cyclopenthyl $\left.) \mathrm{CH}_{2}-\right), 7.76(\mathrm{t}, J=7.1 \mathrm{~Hz}, 1 \mathrm{H}, \mathrm{H}-9)$, $7.86(\mathrm{t}, J=7.0 \mathrm{~Hz}, 1 \mathrm{H}, \mathrm{H}-8), 8.02(\mathrm{~d}, J=8.0 \mathrm{~Hz}, 1 \mathrm{H}, \mathrm{H}-7)$, 8.45 (d, $J=7.7 \mathrm{~Hz}, 1 \mathrm{H}, \mathrm{H}-10), 9.39$ (s, 1H, H-5), 11.75 (s, $1 \mathrm{H},-\mathrm{COOH}$ ). LC-MS $m / z=311$ [M+1]; Anal. Calcd. for $\mathrm{C}_{14} \mathrm{H}_{14} \mathrm{~N}_{4} \mathrm{O}_{2}$ : C, 65.79; H, 5.85; N, 18.05; Found: C, 65.84; $\mathrm{H}, 5.91 ; \mathrm{N}, 18.11$.

2.3 General Method for the Synthesis of Amides of $([1,2,4]$ Triazolo $[1,5-c]$ quinazolin-2-yl)alkylcarboxilic Acids $(4 \mathbf{a}-\mathbf{j})$

Method A. 5.5 of mmol of para-methoxybenzylamine and 1-2 $\mathrm{mL}$ of DMF was added to the $5 \mathrm{mmol}$ of corresponding ester (3a, $\mathbf{3} \mathbf{b})$. The formed mixture was treated at $140-150{ }^{\circ} \mathrm{C}$ for $3-4 \mathrm{~h}$. The $5 \mathrm{~mL}$ of methanol and $5 \mathrm{~mL}$ of water were added to the mixture after completing of the reaction. The formed precipitate was filtered off and dried. Obtained compounds may be additionally purified by crystallization from ethanol.

Method B. $0.89 \mathrm{~g}(5.5 \mathrm{mmol})$ of $N, N^{\prime}$-carbonyldiimidazole (CDI) was added to the solution of $5 \mathrm{mmol}$ of corresponding carboxylic acid (3a-f) in $20 \mathrm{~mL}$ of anhydrous dioxane. The formed mixture was heated at $80{ }^{\circ} \mathrm{C}$ for $1 \mathrm{~h}$ (until the carbon dioxide was completely released). Then 5 mmol of corresponding amine was added and stirred (or refluxed) for $1.5-3 \mathrm{~h}$. The formed mixture was cooled and poured into water and acidified by hydrochloric acid to $\mathrm{pH}$ 5-6. The formed mixture was filtered off and dried.

$N$-(4-Methoxybenzyl)-[1,2,4] triazolo[1,5-c]quinazoline-2-carboxamide (4a). Yield: $0.97 \mathrm{~g}(58 \%)$. Mp 183-185 ${ }^{\circ} \mathrm{C}$; IR $3857\left(v_{\mathrm{NH}}\right), 3753\left(v_{\mathrm{NH}}\right), 2928\left(v_{\mathrm{CH} 2}\right), 2510,1656$ $\left(v_{\mathrm{CO}}\right), 1553\left(\delta_{\mathrm{NH}}\right), 1516,1465,1319,1236,741,689 \mathrm{~cm}^{-1}$. ${ }^{1} \mathrm{H}$ NMR $\left(400 \mathrm{MHz}\right.$, DMSO- $\left.d_{6}\right) \delta 3.74\left(\mathrm{~s}, 3 \mathrm{H},-\mathrm{OC}_{3}\right)$, $4.47\left(\mathrm{~d}, J=5.7 \mathrm{~Hz}, 2 \mathrm{H},-\mathrm{NHC}_{2_{2}}\right), 6.81(\mathrm{~d}, J=7.6 \mathrm{~Hz}, 2 \mathrm{H}$, H-3,5 Bn), 7.29 (d, $J=7.9 \mathrm{~Hz}, 2 \mathrm{H}, \mathrm{H}-2,6 \mathrm{Bn}), 7.81(\mathrm{t}, J=$ $7.6 \mathrm{~Hz}, 1 \mathrm{H}, \mathrm{H}-9), 7.92(\mathrm{t}, J=7.8 \mathrm{~Hz}, 1 \mathrm{H}, \mathrm{H}-8), 8.07(\mathrm{~d}, J=$ $7.9 \mathrm{~Hz}, 1 \mathrm{H}, \mathrm{H}-7), 8.49(\mathrm{~d}, J=8.0 \mathrm{~Hz}, 1 \mathrm{H}, \mathrm{H}-10), 9.06(\mathrm{t}, J=$ $\left.5.4 \mathrm{~Hz}, 1 \mathrm{H},-\mathrm{NHCH}_{2}-\right), 9.56(\mathrm{~s}, 1 \mathrm{H}, \mathrm{H}-5)$. LC-MS $\mathrm{m} / z=$ $334[\mathrm{M}+1]$; Anal. Calcd. for $\mathrm{C}_{18} \mathrm{H}_{15} \mathrm{~N}_{5} \mathrm{O}_{2}$ : C, 64.86; $\mathrm{H}$, 4.54; N, 21.01; Found: C, 64.93; H, 4.60; N, 21.09.

2-([1,2,4] Triazolo[ $1,5-c]$ quinazolin-2-yl)- $\mathrm{N}$-(4-methoxybenzyl)acetamide (4b). Yield: $1.20 \mathrm{~g}(69 \%)$. Mp $172-175^{\circ} \mathrm{C}$; IR $3697\left(v_{\mathrm{NH}}\right), 2920\left(v_{\mathrm{CH} 2}\right), 2851\left(v_{\mathrm{CH}}\right), 1669$ $\left(v_{\mathrm{CO}}\right), 1547\left(\delta_{\mathrm{NH}}\right), 1458,1363,1201,1019,862,780,708$, $654 \mathrm{~cm}^{-1} .{ }^{1} \mathrm{H}$ NMR $\left(400 \mathrm{MHz}, \mathrm{DMSO}-d_{6}\right) \delta 3.77(\mathrm{~s}, 3 \mathrm{H}$, $\left.-\mathrm{OC} \underline{H}_{3}\right), 4.45\left(\mathrm{~d}, J=5.7 \mathrm{~Hz}, 2 \mathrm{H},-\mathrm{NHC}_{2_{2}}\right), 6.87(\mathrm{~d}, J=7.6$ $\mathrm{Hz}, 2 \mathrm{H}, \mathrm{H}-3,5 \mathrm{Bn}), 7.22$ (d, $J=7.9 \mathrm{~Hz}, 2 \mathrm{H}, \mathrm{H}-2,6 \mathrm{Bn}), 7.63$ $(\mathrm{t}, J=7.6 \mathrm{~Hz}, 1 \mathrm{H}, \mathrm{H}-9), 7.78(\mathrm{t}, J=7.8 \mathrm{~Hz}, 1 \mathrm{H}, \mathrm{H}-8), 8.00$ $(\mathrm{d}, J=7.9 \mathrm{~Hz}, 1 \mathrm{H}, \mathrm{H}-7), 8.43(\mathrm{~d}, J=8.1 \mathrm{~Hz}, 1 \mathrm{H}, \mathrm{H}-10), 9.08$ $\left.\left(\mathrm{t}, J=5.4 \mathrm{~Hz}, 1 \mathrm{H},-\mathrm{NHCH}_{2}\right)^{-}\right), 9.38(\mathrm{~s}, 1 \mathrm{H}, \mathrm{H}-5)$. LC-MS $m / z=348[\mathrm{M}+1]$; Anal. Calcd. for $\mathrm{C}_{19} \mathrm{H}_{17} \mathrm{~N}_{5} \mathrm{O}_{2}$ : C, 65.69; H, 4.93; N, 20.16; Found: C, 65.74; H, 4.98; N, 20.21.

3-([1,2,4]Triazolo[1,5-c]quinazolin-2-yl)- $\mathrm{N}$-(4-fluorophenyl)propanamide (4c). Yield: $1.07 \mathrm{~g}(64 \%)$. Mp 206-208 ${ }^{\circ} \mathrm{C}$; IR $3297\left(v_{\mathrm{NH}}\right), 1665\left(v_{\mathrm{CO}}\right), 1530\left(\delta_{\mathrm{NH}}\right), 1493,1371,1214$, $901,834,767,710 \mathrm{~cm}^{-1} .{ }^{1} \mathrm{H}$ NMR (400 MHz, DMSO- $\left.d_{6}\right) \delta$ $2.90\left(\mathrm{t}, J=7.6 \mathrm{~Hz}, 2 \mathrm{H},-\mathrm{CH}_{2} \mathrm{CH}_{2^{-}}\right), 3.54-3.07(\mathrm{t}, J=7.6 \mathrm{~Hz}$, $\left.2 \mathrm{H},-\mathrm{CH}_{2} \mathrm{CH}_{2}-\right), 6.95(\mathrm{t}, J=8.6 \mathrm{~Hz}, 2 \mathrm{H}, \mathrm{H}-3,5 \mathrm{Ph}), 7.60$ (dd, $\left.J^{2}=8.5 \mathrm{~Hz}, J^{3}=4.9 \mathrm{~Hz}, 2 \mathrm{H}, \mathrm{H}-2,6 \mathrm{Ph}\right), 7.74(\mathrm{t}, J=7.5 \mathrm{~Hz}, 1 \mathrm{H}$, H-9), $7.84(\mathrm{t}, J=7.7 \mathrm{~Hz}, 1 \mathrm{H}, \mathrm{H}-8), 7.99(\mathrm{~d}, J=8.1 \mathrm{~Hz}, 1 \mathrm{H}$, $\mathrm{H}-7), 8.40(\mathrm{~d}, J=8.3 \mathrm{~Hz}, 1 \mathrm{H}, \mathrm{H}-10), 9.95(\mathrm{~s}, 1 \mathrm{H},-\mathrm{NH}-)$. LCMS $m / z=336[\mathrm{M}+1]$; Anal. Calcd. for $\mathrm{C}_{18} \mathrm{H}_{14} \mathrm{FN}_{5} \mathrm{O}: \mathrm{C}$, 64.47; H, 4.21; N, 20.88; Found: C, 64.54; H, 4.26; N, 20.93.

3-([1,2,4]Triazolo[1,5-c]quinazolin-2-yl)- $\mathrm{N}$-(4-bromophenyl)propanamide (4d). Yield: $0.93 \mathrm{~g}$ (47\%). Mp 210-212 ${ }^{\circ} \mathrm{C}$; IR $3840\left(v_{\mathrm{NH}}\right), 3727\left(v_{\mathrm{NH}}\right), 3286\left(v_{\mathrm{NH}}\right), 1662$ $\left(v_{\mathrm{CO}}\right), 1529\left(\delta_{\mathrm{NH}}\right), 1488,1394,1245,900,767,711,660$ $\mathrm{cm}^{-1} .{ }^{1} \mathrm{H}$ NMR $\left(400 \mathrm{MHz}, \mathrm{DMSO}-d_{6}\right) \delta 2.91(\mathrm{t}, J=7.6 \mathrm{~Hz}$, $\left.2 \mathrm{H},-\mathrm{CH}_{2} \underline{\mathrm{C}}_{2}-\right), 3.24\left(\mathrm{t}, J=7.6 \mathrm{~Hz}, 2 \mathrm{H},-\mathrm{C}_{2} \mathrm{CH}_{2}-\right), 7.32$ $(\mathrm{d}, J=8.7 \mathrm{~Hz}, 2 \mathrm{H}, \mathrm{H}-2,6 \mathrm{Ph}), 7.56(\mathrm{~d}, J=8.6 \mathrm{~Hz}, 2 \mathrm{H}, \mathrm{H}-3,5$ $\mathrm{Ph}), 7.74(\mathrm{t}, J=7.6 \mathrm{~Hz}, 1 \mathrm{H}, \mathrm{H}-9), 7.84(\mathrm{t}, J=7.7 \mathrm{~Hz}, 1 \mathrm{H}$, $\mathrm{H}-8), 8.00(\mathrm{~d}, J=8.2 \mathrm{~Hz}, 1 \mathrm{H}, \mathrm{H}-7), 8.41(\mathrm{~d}, J=8.0 \mathrm{~Hz}, 1 \mathrm{H}$, $\mathrm{H}-10$ ), 9.36 (s, $1 \mathrm{H}, \mathrm{H}-5$ ), 10.03 (s, 1H, -NH-). LC-MS m/z $=396[\mathrm{M}+1]$; Anal. Calcd. for $\mathrm{C}_{18} \mathrm{H}_{14} \mathrm{BrN}_{5} \mathrm{O}: \mathrm{C}, 54.56 ; \mathrm{H}$, 3.56; N, 17.67; Found: C, 54.63; H, 3.62; N, 17.73. 
Ethyl 4-(3-([1,2,4] triazolo[1,5-c]quinazolin-2-yl)propanamido)benzoate (4e). Yield: $1.18 \mathrm{~g}(61 \%)$. Mp 214-216 ${ }^{\circ} \mathrm{C}$; IR $3857\left(v_{\mathrm{NH}}\right), 3725\left(v_{\mathrm{NH}}\right), 2901\left(v_{\mathrm{CH} 2}\right), 1711\left(v_{\mathrm{CO}}\right)$, $1667\left(v_{\mathrm{CO}}\right), 1599\left(\delta_{\mathrm{NH}}\right), 1493,1408,1311,1274,899,854$, $766,694 \mathrm{~cm}^{-1} .{ }^{1} \mathrm{H}$ NMR $\left(400 \mathrm{MHz}, \mathrm{DMSO}-d_{6}\right) \delta 1.35(\mathrm{t}, J$ $\left.=7.1 \mathrm{~Hz}, 3 \mathrm{H},-\mathrm{CH}_{2} \mathrm{C}_{3}\right), 2.96\left(\mathrm{t}, J=7.6 \mathrm{~Hz}, 2 \mathrm{H},-\mathrm{CH}_{2} \mathrm{C}_{2} 2^{-}\right)$, $3.25\left(\mathrm{dd}, J^{2}=8.6 \mathrm{~Hz}, J^{3}=6.7 \mathrm{~Hz}, 2 \mathrm{H},-\mathrm{CH}_{2} \mathrm{CH}_{2}-\right), 4.26(\mathrm{q}$, $\left.J=7.1 \mathrm{~Hz}, 2 \mathrm{H},-\mathrm{CH}_{2} \mathrm{CH}_{3}\right), 7.69(\mathrm{~d}, J=8.4 \mathrm{~Hz}, 2 \mathrm{H}, \mathrm{H}-2,6$ $\mathrm{Ph}$ ), 7.73 (t, $J=7.6 \mathrm{~Hz}, 1 \mathrm{H}, \mathrm{H}-9), 7.88-7.80(\mathrm{~m}, 3 \mathrm{H}, \mathrm{H}-8$, $\mathrm{H}-3,5 \mathrm{Ph}), 7.99(\mathrm{~d}, J=8.2 \mathrm{~Hz}, 1 \mathrm{H}, \mathrm{H}-7), 8.40(\mathrm{~d}, J=7.5 \mathrm{~Hz}$, 1H, H-10), 9.36 (s, 1H, H-5), 10.21 (s, 1H, -NH-). LC-MS $m / z=390[\mathrm{M}+1]$; Anal. Calcd. for $\mathrm{C}_{21} \mathrm{H}_{19} \mathrm{~N}_{5} \mathrm{O}_{3}: \mathrm{C}, 64.77$; H, 4.92; N, 17.98; Found: C, 64.74; H, 4.99; N, 18.03 .

4-([1,2,4] Triazolo[1,5-c] quinazolin-2-yl)- $\mathrm{N}$-(4-fluorophenyl)butanamide (4f). Yield: $0.78 \mathrm{~g}(45 \%)$. Mp 173-175 ${ }^{\circ} \mathrm{C}$; IR $3295\left(v_{\mathrm{NH}}\right), 1658\left(v_{\mathrm{CO}}\right), 1528\left(\delta_{\mathrm{NH}}\right), 1504,1432$, 1404, 1336, 1207, 904, 835, 722, $697 \mathrm{~cm}^{-1} .{ }^{1} \mathrm{H}$ NMR $(400$ $\left.\mathrm{MHz}, \mathrm{DMSO}-d_{6}\right) \delta 2.18\left(\mathrm{p}, J=7.3 \mathrm{~Hz}, 2 \mathrm{H},-\mathrm{CH}_{2} \mathrm{CH}_{2} \mathrm{CH}_{2}-\right.$ ), $2.41\left(\mathrm{t}, J=7.4 \mathrm{~Hz}, 2 \mathrm{H},-\left(\mathrm{CH}_{2}\right)_{2} \mathrm{CH}_{2}-\right), 2.97(\mathrm{t}, J=7.7 \mathrm{~Hz}$, $\left.2 \mathrm{H},-\mathrm{CH}_{2}\left(\mathrm{CH}_{2}\right)_{2}-\right), 6.93(\mathrm{t}, J=8.7 \mathrm{~Hz}, 2 \mathrm{H}, \mathrm{H}-3,5 \mathrm{Ph}), 7.56$ $\left(\mathrm{dd}, J^{2}=8.8 \mathrm{~Hz}, J^{3}=5.0 \mathrm{~Hz}, 2 \mathrm{H}, \mathrm{H}-2,6 \mathrm{Ph}\right), 7.75(\mathrm{t}, J=7.4$ $\mathrm{Hz}, 1 \mathrm{H}, \mathrm{H}-9$ ), 7.85 (t, $J=7.7 \mathrm{~Hz}, 1 \mathrm{H}, \mathrm{H}-7$ ), 8.01 (d, $J=8.2$ $\mathrm{Hz}, 1 \mathrm{H}, \mathrm{H}-8), 8.43$ (d, J = 7.8 Hz, 1H, H-10), 9.36 (s, $1 \mathrm{H}$, $\mathrm{H}-5$ ), 9.70 (s, $1 \mathrm{H},-\mathrm{NH}-$ ). LC-MS $m / z=350$ [M+1]; Anal. Calcd. for $\mathrm{C}_{19} \mathrm{H}_{16} \mathrm{FN}_{5} \mathrm{O}$ : C, 65.32; H, 4.62; N, 20.05; Found: C, 65.39; H, 4.69; N, 20.13 .

4-([1,2,4] Triazolo[1,5-c] quinazolin-2-yl)- $N$-(4-chlorophenyl)butanamide (4g). Yield: $0.96 \mathrm{~g}$ (52\%). Mp 199-201 ${ }^{\circ} \mathrm{C}$; IR $3904\left(v_{\mathrm{NH}}\right), 3725\left(v_{\mathrm{NH}}\right), 3249\left(v_{\mathrm{NH}}\right), 1656$ $\left(v_{\mathrm{CO}}\right), 1520\left(\delta_{\mathrm{NH}}\right), 1491,1396,1333,1251,903,817,771$, $721,702,668 \mathrm{~cm}^{-1} .{ }^{1} \mathrm{H}$ NMR $\left(400 \mathrm{MHz}, \mathrm{DMSO}-d_{6}\right) \delta$ 2.25-2.07 (m, $\left.2 \mathrm{H},-\mathrm{CH}_{2} \mathrm{CH}_{2} \mathrm{CH}_{2}-\right), 2.43(\mathrm{t}, J=7.2 \mathrm{~Hz}, 2 \mathrm{H}$, $\left.-\left(\mathrm{CH}_{2}\right)_{2} \mathrm{CH}_{2}-\right), 2.98\left(\mathrm{t}, J=7.3 \mathrm{~Hz}, 2 \mathrm{H},-\mathrm{CH}_{2}\left(\mathrm{CH}_{2}\right)_{2}-\right), 7.17$ $(\mathrm{d}, J=8.8 \mathrm{~Hz}, 2 \mathrm{H}, \mathrm{H}-2,6 \mathrm{Ph}), 7.57(\mathrm{~d}, J=8.8 \mathrm{~Hz}, 2 \mathrm{H}$, $\mathrm{H}-3,5 \mathrm{Ph}), 7.74(\mathrm{t}, J=7.5 \mathrm{~Hz}, 1 \mathrm{H}, \mathrm{H}-9), 7.84(\mathrm{t}, J=8.4 \mathrm{~Hz}$, $1 \mathrm{H}, \mathrm{H}-8), 8.00(\mathrm{~d}, J=7.7 \mathrm{~Hz}, 1 \mathrm{H}, \mathrm{H}-7), 8.41(\mathrm{~d}, J=9.1 \mathrm{~Hz}$, 1H, H-10), 9.35 (s, 1H, H-5), 9.80 (s, 1H, -NH-). LC-MS $m / z=366[\mathrm{M}+1]$; Anal. Calcd. for $\mathrm{C}_{19} \mathrm{H}_{16} \mathrm{ClN}_{5} \mathrm{O}: \mathrm{C}$, 62.38; H, 4.41; N, 19.14; Found: C, 62.46; H, 4.47; N, 19.19 .

4-([1,2,4]Triazolo $[1,5-c]$ quinazolin-2-yl)- $N$-(4-bromophenyl)butanamide (4h). Yield: $1.41 \mathrm{~g}(69 \%)$. Mp 200-202 ${ }^{\circ} \mathrm{C}$; IR $3250\left(v_{\mathrm{NH}}\right), 1651\left(v_{\mathrm{CO}}\right), 1522\left(\delta_{\mathrm{NH}}\right), 1489$, 1334, 1283, 1244, 904, 814, 773, 703, $659 \mathrm{~cm}^{-1} .{ }^{1} \mathrm{H}$ NMR $\left(400 \mathrm{MHz}, \mathrm{DMSO}-d_{6}\right) \delta 2.18(\mathrm{p}, J=7.4 \mathrm{~Hz}, 2 \mathrm{H}$, $\left.-\mathrm{CH}_{2} \mathrm{CH}_{2} \mathrm{CH}_{2}-\right), 2.42\left(\mathrm{t}, J=7.3 \mathrm{~Hz}, 2 \mathrm{H},-\left(\mathrm{CH}_{2}\right)_{2} \mathrm{CH}_{2}-\right)$, $2.97\left(\mathrm{t}, J=7.5 \mathrm{~Hz}, 2 \mathrm{H},-\mathrm{CH}_{2}\left(\mathrm{CH}_{2}\right)_{2}-\right), 7.31(\mathrm{~d}, J=8.8 \mathrm{~Hz}$, $2 \mathrm{H}, \mathrm{H}-2,6 \mathrm{Ph}$ ), 7.52 (d, $J=8.7 \mathrm{~Hz}, 2 \mathrm{H}, \mathrm{H}-3,5 \mathrm{Ph}), 7.75$ (t, $=7.5 \mathrm{~Hz}, 1 \mathrm{H}, \mathrm{H}-9), 7.85(\mathrm{t}, J=8.3 \mathrm{~Hz}, 1 \mathrm{H}, \mathrm{H}-8), 8.00(\mathrm{~d}, J$ $=8.2 \mathrm{~Hz}, 1 \mathrm{H}, \mathrm{H}-7), 8.42(\mathrm{~d}, J=7.9 \mathrm{~Hz}, 1 \mathrm{H}, \mathrm{H}-10), 9.36(\mathrm{~s}$, $1 \mathrm{H}, \mathrm{H}-5), 9.79$ (s, $1 \mathrm{H},-\mathrm{NH}$-). LC-MS $m / z=411[\mathrm{M}+1]$; Anal. Calcd. for $\mathrm{C}_{19} \mathrm{H}_{16} \mathrm{BrN}_{5} \mathrm{O}$ : C, 55.62; $\mathrm{H}, 3.93 ; \mathrm{N}, 17.07$; Found: C, 55.68; H, 4.01; N, 17.12.
Ethyl 4-(4-([1,2,4] triazolo[1,5-c]quinazolin-2-yl)butanamido)benzoate (4i). Yield: $0.77 \mathrm{~g}(38 \%)$. Mp $92-94{ }^{\circ} \mathrm{C}$; IR $3340\left(v_{\mathrm{NH}}\right), 1692\left(v_{\mathrm{CO}}\right), 1625\left(v_{\mathrm{CO}}\right), 1524\left(\delta_{\mathrm{NH}}\right), 1367$, 1308, 1275, 1017, 960, 901, 854, 767, $697 \mathrm{~cm}^{-1} .{ }^{1} \mathrm{H} \mathrm{NMR}$ $\left(400 \mathrm{MHz}, \mathrm{DMSO}-d_{6}\right) \delta 1.35\left(\mathrm{t}, J=7.1 \mathrm{~Hz}, 3 \mathrm{H},-\mathrm{CH}_{2} \mathrm{C}_{3}\right)$, 2.19 (p, $\left.J=7.3 \mathrm{~Hz}, 2 \mathrm{H},-\mathrm{CH}_{2} \mathrm{C}_{2} \mathrm{CH}_{2^{-}}\right), 2.45(\mathrm{t}, J=6.9 \mathrm{~Hz}$, $\left.2 \mathrm{H},-\left(\mathrm{CH}_{2}\right)_{2} \mathrm{CH}_{2}{ }^{-}\right), 2.99\left(\mathrm{t}, J=7.3 \mathrm{~Hz}, 2 \mathrm{H},-\mathrm{CH}_{2}\left(\mathrm{CH}_{2}\right)_{2}-\right.$ ), $4.26\left(\mathrm{q}, J=7.0 \mathrm{~Hz}, 2 \mathrm{H},-\mathrm{C}_{2} \mathrm{CH}_{3}\right), 7.66(\mathrm{~d}, J=8.3 \mathrm{~Hz}, 2 \mathrm{H}$, $\mathrm{H}-3,5 \mathrm{Ph}), 7.74(\mathrm{t}, J=7.5 \mathrm{~Hz}, 1 \mathrm{H}, \mathrm{H}-9), 7.90-7.79(\mathrm{~m}, 3 \mathrm{H}$, $\mathrm{H}-8, \mathrm{H}-2,6 \mathrm{Ph}), 8.00(\mathrm{~d}, J=8.3 \mathrm{~Hz}, 1 \mathrm{H}, \mathrm{H}-7), 8.42(\mathrm{~d}, J=$ $7.9 \mathrm{~Hz}, 1 \mathrm{H}, \mathrm{H}-10), 9.35$ (s, 1H, H-5), 10.01 (s, 1H, -NH-). LC-MS $m / z=404$ [M+1]; Anal. Calcd. for $\mathrm{C}_{22} \mathrm{H}_{21} \mathrm{~N}_{5} \mathrm{O}_{3}$ : C, 65.50; H, 5.25; N, 17.36; Found: C, 65.57; H, 5.31; N, 17.41 .

4-([1,2,4] Triazolo[1,5-c] quinazolin-2-yl)- $N$-(4-chlorophenyl)-3-methylbutanamide (4j). Yield: $0.59 \mathrm{~g}(31 \%)$. Mp 196-198 ${ }^{\circ} \mathrm{C}$; IR $3348\left(v_{\mathrm{NH}}\right), 1657\left(v_{\mathrm{CO}}\right), 1527\left(\delta_{\mathrm{NH}}\right)$, $1491,1465,1338,1283,1250,903,821,773,703,658 \mathrm{~cm}^{-1}$. ${ }^{1} \mathrm{H}$ NMR $\left(400 \mathrm{MHz}\right.$, DMSO- $\left.d_{6}\right) \delta 1.05(\mathrm{~d}, J=6.6 \mathrm{~Hz}, 3 \mathrm{H}$, $\left.-\mathrm{CH}_{2} \mathrm{CH}\left(\mathrm{C}_{3}\right) \mathrm{CH}_{2}-\right), 2.26\left(\mathrm{dd}, J^{2}=14.2 \mathrm{~Hz}, J^{3}=8.2 \mathrm{~Hz}\right.$, $\left.1 \mathrm{H},-\mathrm{CH}_{2} \mathrm{CH}\left(\mathrm{CH}_{3}\right) \mathrm{C}_{2}{ }^{-}\right), 2.44\left(\mathrm{dd}, 1 \mathrm{H}, J^{2}=14.2 \mathrm{~Hz}, J^{3}=\right.$ $\left.8.2 \mathrm{~Hz},-\mathrm{CH}_{2} \mathrm{CH}\left(\mathrm{CH}_{3}\right) \mathrm{C}_{2}{ }^{-}\right), 2.65\left(\mathrm{dq}, J^{2}=13.8 \mathrm{~Hz}, J^{3}=\right.$ $\left.6.6 \mathrm{~Hz}, 1 \mathrm{H},-\mathrm{CH}_{2} \mathrm{C} \underline{\mathrm{H}}\left(\mathrm{CH}_{3}\right) \mathrm{CH}_{2}-\right), 2.84\left(\mathrm{dd}, J^{2}=14.2 \mathrm{~Hz}, J^{3}\right.$ $\left.\left.=7.8 \mathrm{~Hz}, 1 \mathrm{H},-\underline{\mathrm{C}}_{2} \mathrm{CH}\left(\mathrm{CH}_{3}\right) \mathrm{CH}_{2}\right)^{-}\right), 2.98\left(\mathrm{dd}, J^{2}=14.2 \mathrm{~Hz}\right.$, $\left.J^{3}=6.2 \mathrm{~Hz}, 1 \mathrm{H},-\underline{\mathrm{C}}_{2} \mathrm{CH}\left(\mathrm{CH}_{3}\right) \mathrm{CH}_{2}-\right), 7.16(\mathrm{~d}, J=8.7 \mathrm{~Hz}$, $2 \mathrm{H}, \mathrm{H}-3,5 \mathrm{Ph}), 7.58(\mathrm{~d}, J=8.7 \mathrm{~Hz}, 2 \mathrm{H}, \mathrm{H}-2,6 \mathrm{Ph}), 7.74(\mathrm{t}, J$ $=7.6 \mathrm{~Hz}, 1 \mathrm{H}, \mathrm{H}-9), 7.85(\mathrm{t}, J=7.7 \mathrm{~Hz}, 1 \mathrm{H}, \mathrm{H}-8), 8.00(\mathrm{~d}, J$ $=8.1 \mathrm{~Hz}, 1 \mathrm{H}, \mathrm{H}-7), 8.43(\mathrm{~d}, J=7.9 \mathrm{~Hz}, 1 \mathrm{H}, \mathrm{H}-10), 9.35(\mathrm{~s}$, $1 \mathrm{H}, \mathrm{H}-5), 9.84(\mathrm{~s}, 1 \mathrm{H},-\mathrm{NH}-)$. LC-MS $m / z=380[\mathrm{M}+1]$; Anal. Calcd. for $\mathrm{C}_{20} \mathrm{H}_{18} \mathrm{ClN}_{5} \mathrm{O}$ : C, 63.24; $\mathrm{H}, 4.78 ; \mathrm{N}, 18.44$; Found: $\mathrm{C}, 63.31 ; \mathrm{H}, 4.83 ; \mathrm{N}, 18.48$.

\section{2. Anti-inflammatory Activity}

Evaluation of anti-inflammatory activity of the synthesized compounds was conducted on 144 Wistar white rats (weight 150-160 g), obtained from the nursery «Institute of Pharmacology and Toxicology of Ukraine» (Kyiv). All experimental procedures and treatment were carried out according to the European Convention and «Regulations on the use of animals in biomedical research».22 Screening of the synthesized compounds with estimated anti-inflammatory activity began with the study of their effect on exudative phase of acute aseptic inflammation («carrageenan» test). ${ }^{23}$ Phlogogen (1\% aqueous solution of $\lambda$-carrageenan) was subplantally injected in a dose of 0.1 $\mathrm{mL}$ in the rats' back right paw. The left one was used as a control. Intragastric administration of the studied compounds was conducted using atraumatic probe as water solution or finely dispersed suspension stabilized by Tween- 80 in a dose of $10 \mathrm{mg} / \mathrm{kg} 1 \mathrm{~h}$ before the injection of phlogogen. The reference drug diclofenac sodium was administered intragastrically in a recommended dose of 8 $\mathrm{mg} / \mathrm{kg}$ for pre-clinical studies. Measurement of paws volume was conducted before the experiment and in $4 \mathrm{~h}$ («carrageenan» test) after injection of phlogogen using the 
described methods. The activity of these substances was determined by their ability to reduce the swelling compared with control group and was expressed in percentage. It showed how the substance inhibited phlogogen swelling in relation to control swelling where the value was taken as $100 \%$. The activity of the studied compounds was calculated as following:

$$
A A, \%=100 \%-\left(\frac{V p e-V h e}{V p c-V h c} * 100 \%\right)
$$

where $\mathrm{AA}$ - antiexudative activity, $\% ; V_{\mathrm{pe}}$ - the volume of paw edema in the experiment; $V_{\text {he }}$ - the volume of healthy paw in the experiment; $V_{\mathrm{pc}}$ - the volume of paw edema in control; $V_{\mathrm{hc}}$ - the volume of healthy paw in control.

Statistical data processing was performed using a license program «STATISTICA ${ }^{\circledR}$ forWindows 10.0» (StatSoftInc., № AXXR712D833214FAN5) and «SPSS 16.0», «Microsoft Office Excel 360». The results are presented as mean \pm standard error of the mean. Arithmetic mean and standard error of the mean were calculated for each of the studied parameters. During verification of statistical hypothesis, null hypothesis was declined if statistical criterion was $p<0.05 .^{24}$

\section{Results and Discussion}

Previously heterocyclization of corresponding (3H-quinazoline-4-ylidene)hydrazides was described as the most efficient and convenient method for 2-R-[1,2,4] triazolo[ $1,5-c]$ quinazolines synthesis. ${ }^{1-8}$ The preparation of the above-mentioned hydrazides is based on the acylation of 4-hydrazinoquinazoline by anhydrides, acyl halides, $\mathrm{N}$-acyl imidazolides and other highly reactive derivatives of carboxylic acids. ${ }^{1-3}$ Namely, above-mentioned approaches were used for the synthesis of target compounds. It was found that initial compound 1 a may be easily acylated in dioxane medium by ethyl 2-chloro-2-oxoacetate or ethyl 3-chloro-3-oxopropanoate (Method B) as well as by imidazolides of monoethyl esters of oxalic or malonic acids. Above-mentioned rection yielded corresponding hydrazides (2a, $\mathbf{2 b}$, Scheme 1). It should be noted that acylation by acyl halides required the presence of an organic base (triethylamine) and cooling of reaction medium to $0-5{ }^{\circ} \mathrm{C}$. At the same time the reaction between 1a and corresponding imidazolides may be conducted under heating $\left(80^{\circ} \mathrm{C}\right)$. Hydrazides $\mathbf{2} \mathbf{c}-\mathbf{f}$, that contain prolongated alkyl moiety, were synthesized by interaction between initial compound 1a and cyclic anhydrides. Reaction was conducted in dioxane medium at ambient temperature or under heating (Method C, Scheme 1). The significant differences in yield values depending on the synthetic protocols used were not observed.

The following cyclization of hydrazides $2 \mathbf{a}-\mathbf{f}$ yielded corresponding 2-([1,2,4]triazolo[1,5-c]quinazoline-2-yl) carboxylic acids and their esters ( $\mathbf{3 a}-\mathbf{f}$, Scheme 1$)$. Besides, for compounds $\mathbf{3 a}-\mathbf{f}$ one-pot synthesis method was elaborated. Thus compounds $\mathbf{3} \mathbf{a}$ and $\mathbf{3 b}$ were obtained via interaction of 4-hydrazinoquinazoline (1a) with above-mentioned acylhalides in acetic acid medium and the presence of sodium acetate at $0-5{ }^{\circ} \mathrm{C}$ followed by refluxing of reaction mixture for $3 \mathrm{~h}$ (Scheme 1). Compounds $\mathbf{3 d}-\mathbf{f}$ were synthesized by reaction of compound 1a with cyclic anhy-

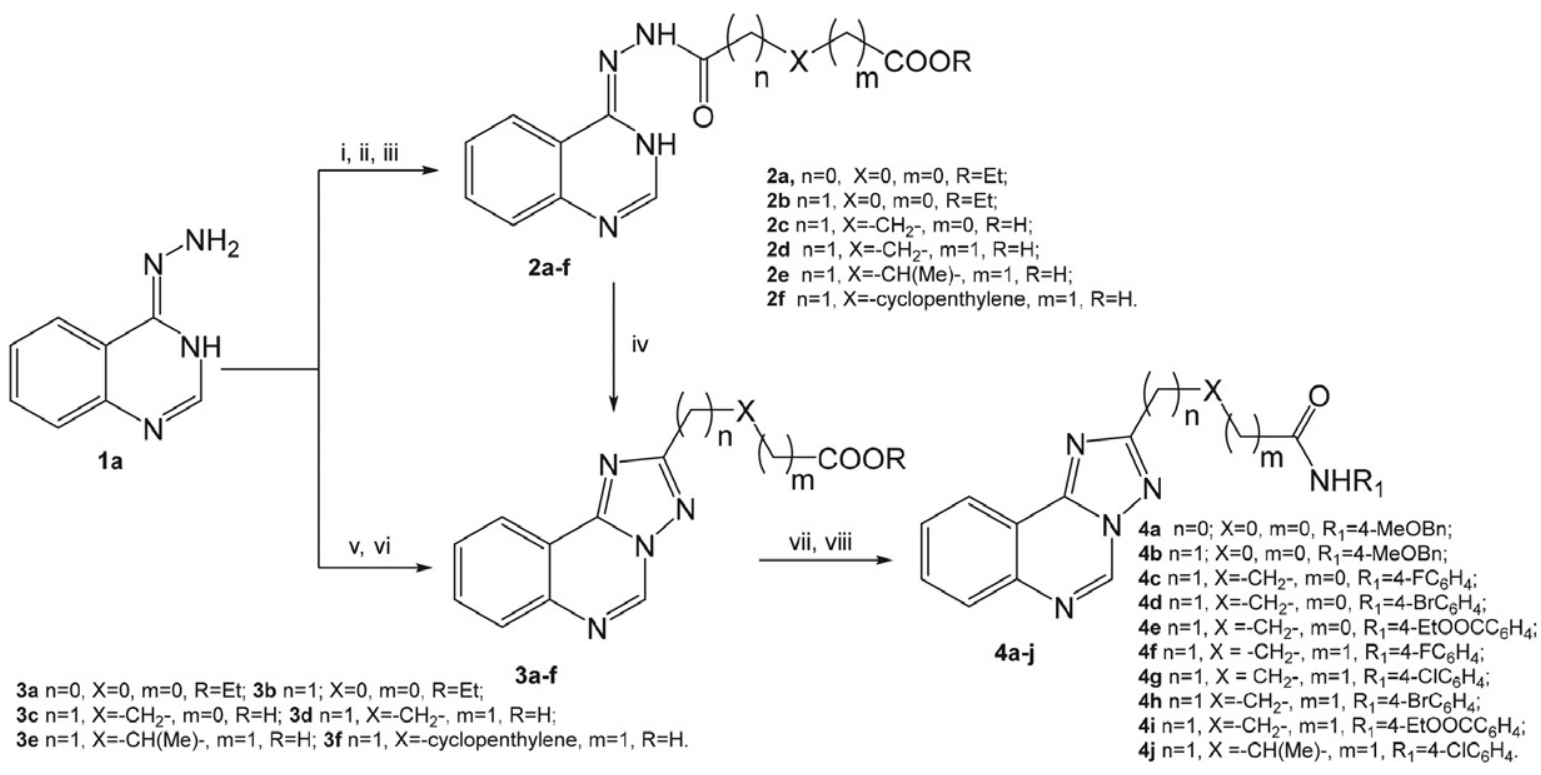

i: $\mathrm{CIC}(\mathrm{O})\left(\mathrm{CH}_{2}\right)_{\mathrm{n}} \mathrm{COOEt}$, dioxane, $\mathrm{N}(\mathrm{Et})_{3}, 0-5^{\circ} \mathrm{C}, 1.5 \mathrm{~h}$; ii: 1) $\mathrm{HCOO}\left(\mathrm{CH}_{2}\right) \mathrm{nCOOEt}$, CDI, dioxane, $80^{\circ} \mathrm{C}, 1.5-3 \mathrm{~h}$; iii: anhydrides, dioxane, reflux, $80^{\circ} \mathrm{C}$, $1-1.5 \mathrm{~h}$ or r.t. $24 \mathrm{~h}$; iv: $\mathrm{AcOH}$, reflux, 3-4 h; v: 1) $\left.\mathrm{ClC}(\mathrm{O})\left(\mathrm{CH}_{2}\right) \mathrm{nCOOEt}, \mathrm{AcOH}, \mathrm{AcONa}, 0-5^{\circ} \mathrm{C}, 1.5 \mathrm{~h} ; 2\right)$ reflux, $3 \mathrm{~h}$ : vi: anhydrides, $\mathrm{AcOH}$, reflux, 3-4 h; vii: 4-MeOBnNH, $1-2$ drop, DMF, $130-140^{\circ} \mathrm{C}, 3-4 \mathrm{~h}$; viii: 1) dioxane, $\left.\mathrm{CDI} .80^{\circ} \mathrm{C} ; 2\right) \mathrm{ArNH}_{2}$ reflux, $1.5 \mathrm{~h}$.

Scheme 1.

Krasovska et al.: Quinazoline-containing Hydrazydes ... 
drides in acetic acid. It should be noted that $[1,2,4]$ triazolo[4,3-c]quinazolines played a role of intermediate products of condensation process. Above-mentioned intermediates underwent acid catalyzed Dimroth-type rearrangement that yielded isomeric $[1,5-c]$-series. ${ }^{1-3}$

Considering the presence of carboxylic or ester group in the structure of compounds 3 it was decided to conduct the chemical modification of above-mentioned fragment to obtain agents with higher anti-inflammatory activity. The synthesis of amides $\mathbf{4}$ was conducted by known methods, namely via aminolysis of esters $\mathbf{3 a}$, $\mathbf{3} \mathbf{b}$ or imidazolides of acids $\mathbf{3} \mathbf{c}-\mathbf{f}$. Compounds $\mathbf{4 a}$ and $\mathbf{4 b}$ were obtained by fusing of initial esters $\mathbf{3} \mathbf{a}$ and $\mathbf{3} \mathbf{b}$ with 4 -methoxybenzylamine at $130-140{ }^{\circ} \mathrm{C}$. At the same time aminolysis of imidazolides of acids $\mathbf{3 c}-\mathbf{f}$ occurred easily in anhydrous dioxane (Scheme 1).

Obtained compounds $\mathbf{2} \mathbf{a}-\mathbf{f}, \mathbf{3 a}-\mathbf{f}, \mathbf{4} \mathbf{a}-\mathbf{j}$ are white, pale yellow crystalline powders that are not soluble in water, soluble in saturated aqueous solution of sodium (potassium) hydrocarbonates (3a-f), alcohols, dioxane and DMF.

Elemental analysis, ${ }^{1} \mathrm{H}$ NMR and LS-MS data proved purity and structure of synthesized substances. The LC-MS using positive-ion atmospheric pressure chemical ionization (APCI) showed the appropriate molecular ions [M+1], which corresponded to the expected molecular weights of 2,3 and 4.

In ${ }^{1} \mathrm{H}$ NMR spectra of hydrazides $\mathbf{2} \mathbf{a}-\mathbf{f}$ the signals of endocyclic NH-protons and protons of hydrazide moiety were observed as broad or doubled singlets at the $11.80-10.88 \mathrm{ppm}$ and 11.11-9.48 ppm, correspondingly. The signals of protons in heterocyclic fragments were registered as a singlet at the 7.91-7.73 ppm (proton at the second position), doublets at the 8.02-7.73 ppm and 7.52-7.36 ppm (protons at the position 5 and positions 7 , correspondingly), triplets at the 7.26-7.19 ppm and 7.17-7.08 ppm (protons at the position 6 and position 8, correspondingly). It should be mentioned that in some cases above-mentioned signals were broadened due to the hydrazide-hydrazonol tautomerism.

${ }^{1} \mathrm{H}$ NMR spectra of compounds $\mathbf{3} \mathbf{a}-\mathbf{f}$ and $\mathbf{4 a} \mathbf{a}-\mathbf{j}$ were characterized by the paramagnetic shift (relative to the ${ }^{1} \mathrm{H}$ NMR spectra of compounds $2 \mathbf{a}-\mathbf{f}$ ) of the signals of the protons in heterocyclic moiety. Above-mentioned phenomenon may be explained by formation of electron-deficient heterocyclic system. The signal of proton at the position 5 of triazoloquinazoline system was characteristic for ${ }^{1} \mathrm{H}$ NMR spectra of compounds $\mathbf{3 a}-\mathbf{f}$ and was registered as a singlet at the 9.56-9.26 $\mathrm{ppm}^{.{ }^{-3}}$ The other protons of tricyclic fragment formed ABCD system which consisted of sequentially located doublets and triplets with corresponding splitting constants.

The signal of carboxylic group protons was not observed in ${ }^{1} \mathrm{H}$ NMR spectra of compounds $\mathbf{2 c}-\mathbf{f}$ due to the deuterium exchanging processes. At the same time the signal of above-mentioned group protons was registered in low field as singlets at the $11.90-11.75 \mathrm{ppm}$ in ${ }^{1} \mathrm{H}$ NMR spectra of compounds $\mathbf{3 c}-\mathbf{f}$. In ${ }^{1} \mathrm{H}$ NMR spectra of compounds $4 \mathbf{a}-\mathbf{j}$ the chemical shifts of the signals of amide group proton depended on its chemical surrounding and were registered as triplets at the 9.08-9.06 ppm (compounds $4 \mathbf{a}, \mathbf{4 b})$ or singlets at the $10.21-9.70 \mathrm{ppm}(\mathbf{4} \mathbf{c}-\mathbf{j})$. Besides, the signals of aromatic protons of benzylamide or anilide fragments were characteristic for ${ }^{1} \mathrm{H}$ NMR spectra of compounds $4 .{ }^{25}$ In ${ }^{1} \mathrm{H}$ NMR spectra of compounds 2,3 and 4 the signals of aliphatic moieties protons were observed with corresponding chemical shifts and multiplicity. ${ }^{25}$ It should be noted that additional splitting of signals caused by diastereotopic methylene group protons of 3-methylbutyl fragment was observed in ${ }^{1} \mathrm{H}$ NMR spectra of compounds $\mathbf{3 e}$ and $\mathbf{4 j}$.

The characteristic bands of stretching vibrations of $\mathrm{NH}$ group at the $3256-3007 \mathrm{~cm}^{-1}$, CO group at the $1741-1703 \mathrm{~cm}^{-1}$, CONH group ("amide I" band) at the $1689-1600 \mathrm{~cm}^{-1}$, "amide II" band at the $1616-1519 \mathrm{~cm}^{-1}$ were present in IR spectra of compounds 2. IR spectra of compounds 3 were characterized by the absence of absorption bands caused by the stretching vibrations of amide group at the $3256-3007 \mathrm{~cm}^{-1}$ and the presence of intensive bands of CO group stretching vibrations at the 1730-1706 $\mathrm{cm}^{-1}$. IR spectra of compounds 4 were characterized by wide bands of $\mathrm{NH}$ group stretching vibrations at the $1669-1651 \mathrm{~cm}^{-1}$, stretching vibrations bands of $\mathrm{NH}$ group at the $3857-3249 \mathrm{~cm}^{-1}$, vibrations bands of CO group («amide I») at the $1669-1651 \mathrm{~cm}^{-1}$ and combined stretching-deformation vibrations of $\mathrm{NH}$ and $\mathrm{CN}$ group («amide II» band) at the $1599-1520 \mathrm{~cm}^{-1}$. IR spectra of halogen-containing compounds were additionally characterized by absorption bands caused by stretching vibrations of C-halogen bond: $v_{\mathrm{C}-\mathrm{F}}$ at the $1110-1102 \mathrm{~cm}^{-1}(\mathbf{4 c}, 4 \mathrm{f}), v_{\mathrm{C}-\mathrm{Br}}$ at the $660-650 \mathrm{~cm}^{-1}(4 \mathrm{~d}, 4 \mathrm{~h}), v_{\mathrm{C}-\mathrm{Cl}}$ at the $750-700 \mathrm{~cm}^{-1}(\mathbf{4 g}, 4 \mathbf{j})$. It should be noted that in IR spectra of compounds 2,3 and 4 low intensity bands $v_{\mathrm{C}=\mathrm{C}}$ bond at the $1486-1424 \mathrm{~cm}^{-1}$, $\gamma_{(=\mathrm{C}-\mathrm{H})}$ at the $850-666 \mathrm{~cm}^{-1}, v_{\mathrm{CH} 2}$ and $\delta_{\mathrm{CH}_{2}}$-group at the 2988-2928 and 1491-1404 $\mathrm{cm}^{-1}$ were observed.

Screening of obtained compounds for anti-exudative activity was conducted in continuation of our studies aimed to the purposeful search of anti-inflammatory agents among compounds that contain heterocyclic fragment and carboxylic group. The studies were carried out using carrageenan-induced inflammation model. ${ }^{23} \mathrm{Ac}$ cording to the obtained results (Table 1) in most of the cases obtained compounds were characterized by moderate anti-inflammatory activity. It should be noted that pharmacological effects of some compounds were comparable with activity of reference compound - sodium diclofenac. Thus, compounds $4 \mathrm{a}, \mathbf{2 b}, \mathbf{4 e}, \mathbf{4 g}, 4 \mathrm{~h}, 2 \mathrm{e}$ and $4 \mathrm{j}$ revealed anti-inflammatory activity on the level of $40.28-54.86 \%$.

The conducted SAR-analysis showed that anti-exudative activity of hydrazides 2 depends on the length of alkyl moiety between heterocyclic fragment and carboxylic group. Compounds with propyl (2b), 3-methylpentyl (2e) and (cyclopentyl)ethyl (2f) fragments were the most 
Table 1. Anti-inflammatory activity of the synthesized compounds $(\mathrm{M} \pm \mathrm{m}, n=6)^{*}$

\begin{tabular}{ccccc}
\hline № & Compd. & $\begin{array}{c}\text { The healthy paw } \\
\text { volume, } \mathbf{~ m}^{*}\end{array}$ & $\begin{array}{c}\text { Edema paw volume } \\
\text { on } \mathbf{4}^{\text {th }} \mathbf{h} \text { of } \mathbf{e x p} . \mathbf{~ m L}^{*}\end{array}$ & AA, \% \\
\hline & $\begin{array}{c}\text { Control } \\
\text { Diclofenac sodium }\end{array}$ & $1.410 \pm 0.021$ & $2.370 \pm 0.042$ & - \\
1. & $\mathbf{2 a}$ & $1.553 \pm 0.041$ & $1.843 \pm 0.046$ & 69.79 \\
2. & $\mathbf{3 a}$ & $1.336 \pm 0.037$ & $2.186 \pm 0.133$ & 17.01 \\
3. & $\mathbf{4 a}$ & $1.64 \pm 0.039$ & $2.330 \pm 0.066$ & -3.47 \\
4. & $\mathbf{2 b}$ & $1.723 \pm 0.046$ & $2.073 \pm 0.027$ & 54.86 \\
5. & $\mathbf{3 b}$ & $1.460 \pm 0.080$ & $2.273 \pm 0.059$ & 42.71 \\
6. & $\mathbf{4 b}$ & $1.623 \pm 0.044$ & $2.073 \pm 0.099$ & 36.11 \\
7. & $\mathbf{2 c}$ & $1.370 \pm 0.060$ & $2.226 \pm 0.075$ & 37.15 \\
8. & $\mathbf{3 c}$ & $1.280 \pm 0.047$ & $2.100 \pm 0.058$ & 23.96 \\
9. & $\mathbf{4 c}$ & $1.370 \pm 0.026$ & $2.126 \pm 0.108$ & 11.81 \\
10. & $\mathbf{4 d}$ & $1.640 \pm 0.024$ & $2.110 \pm 0.114$ & 22.92 \\
11. & $\mathbf{4 e}$ & $1.460 \pm 0.046$ & $2.140 \pm 0.075$ & 47.92 \\
12. & $\mathbf{2 d}$ & $1.306 \pm 0.053$ & $2.156 \pm 0.076$ & 27.43 \\
13. & $\mathbf{3 d}$ & $1.400 \pm 0.066$ & $2.076 \pm 0.097$ & 19.79 \\
14. & $\mathbf{4 f}$ & $1.870 \pm 0.030$ & $2.333 \pm 0.095$ & 2.78 \\
15. & $\mathbf{4 g}$ & $1.573 \pm 0.048$ & $2.556 \pm 0.075$ & 28.47 \\
16. & $\mathbf{4 h}$ & $1.403 \pm 0.020$ & $1.110 \pm 0.074$ & 44.10 \\
17. & $\mathbf{4 i}$ & $1.740 \pm 0.037$ & $2.430 \pm 0.055$ & 42.36 \\
18. & $\mathbf{2 e}$ & $1.463 \pm 0.081$ & $1.943 \pm 0.110$ & 28.13 \\
19. & $\mathbf{3 e}$ & $1.386 \pm 0.045$ & $2.206 \pm 0.098$ & 50.00 \\
20. & $\mathbf{4 j}$ & $1.563 \pm 0.066$ & $2.136 \pm 0.058$ & 14.58 \\
21. & $\mathbf{2 f}$ & $1.386 \pm 0.049$ & $1.983 \pm 0.122$ & 40.28 \\
22. & $\mathbf{3 f}$ & $1.370 \pm 0.05$ & $2.096 \pm 0.072$ & 37.85 \\
\hline
\end{tabular}

${ }^{*}$ Note: significant changes in control $(p<0.05) ; n$ is the number of animals in the group

active among the compounds $\mathbf{2}$. Compounds 3 were less active comparing to hydrazides 2 . Thus, cyclization of compounds 2 resulted in significant decrease of anti-inflammatory activity. At the same time amides 4 revealed high pharmacological effect. It was shown that level of anti-inflammatory activity depend on the nature of amide fragment. Amides that contain 4-chloro(bromo)phenyl moieties $(\mathbf{4 d}, \mathbf{4 g}, \mathbf{4 h}, \mathbf{4 j})$ showed higher activity comparing to compounds $4 \mathbf{e}$ and $4 \mathbf{i}$ with "pharmacophore" 4-ethylcarboxyphenyl fragment. The presence of 4-methoxybenzylamide moiety (compounds $\mathbf{4 a}$ and $\mathbf{4 b}$ ) also had positive effect on the level of anti-inflammatory activity.

The conducted studies showed that amides of $([1,2,4]$ triazolo[1,5-c] quinazoline-2-yl)alkyl carboxylic acids are promising group of anti-inflammatory agents. The further study of their chemical modification and profound study of their pharmacological effects are reasonable in scope of purposeful search of novel effective anti-inflammatory drugs.

\section{Conclusion}

It was found that acylation of quinazolin-4 $(3 H)$-ylidenehydrazine by cyclic anhydrides of dicarboxylic acids, acylhalides or imidazolides of dicarboxylic acids monoesters is an efficient approach for the synthesis of corresponding hydrazides. The cyclization of obtained hydrazides yielded products that combine $[1,2,4]$ triazolo[1,5-c] quinazoline fragment and carboxylic or ester groups in their structures. Above-mentioned compounds were used for the synthesis of corresponding amides. Screening of the synthesized compounds for anti-exudative activity revealed the potential of $([1,2,4]$ triazolo[ $[1,5-c]$ quinazoline-2-yl)alkyl carboxylic acids amides as promising anti-inflammatory agents.

\section{References}

1. O. V. Karpenko, S. I. Kovalenko, J. Org. Pharm. Chem. 2005, 2, 47-54.

2. O. V. Karpenko, S. I. Kovalenko, J. Org. Pharm. Chem. 2005, 4, 61-69.

3. O. V. Karpenko, S. I. Kovalenko, J. Org. Pharm. Chem. 2006, 2, 65-70.

4. Yu. V. Martynenko, M. S. Kazunin, E. A. Selivanova, S. I. Kovalenko, Zaporozhye Med. J. 2016, 4, 89-96.

5. Yu. V. Martynenko, M. S. Kazunin, I. S. Nosulenko, G. G. Berest, S. I. Kovalenko, O. M. Kamyshnyi, N. M. Polishchuk, Zaporozhye Med. J. 2018, 3, 413-420.

6. Yu. V. Martynenko, O. M. Antypenko, I. S. Nosulenko, G. G. Berest, S. S. Kovalenko, Anti-Inflammatory \& Anti-Allergy Agents in Med. Chem. 2019, 19, 60-71. 
7. Yu. V. Martynenko, O. M. Antypenko, O. A. Brazhko, I. B. Labenska, S. I. Kovalenko, Acta Chim. Slov. 2019, 66, 145-154. DOI:10.17344/acsi.2018.4731

8. L. N. Antypenko, A. V. Karpenko, S. I. Kovalenko, A. M. Katsev, E. Z. Komarovska-Porokhnyavets, V. P. Novikov, Arch. Pharm. Chem. Life Sci. 2009, 342, 651-662.

DOI:10.1002/ardp.200900077

9. S. K. Pandey, A. Singh, A. Singh, A. Nizamuddin, Eur. J. Med. Chem. 2009, 44, 1188-119.

10. J. Kehler, A. Ritzen, M. Langgård, S. L. Petersen, M. M. Farah, C. Bundgaard, J. P. Kilburn, Bioorg. Med. Chem. Lett. 2011, 21, 3738-3742. DOI:10.1016/j.bmcl.2011.04.067

11. S. I. Kovalenko, L. M. Antypenko, A. K. Bilyi, S. V. Kholodnyak, O. V. Karpenko, O. M. Antypenko, N. S. Mykhaylova, T. I. Los, O. S. Kolomoets, Sci. Pharm. 2013, 81, 359-391.

12. V. G. Ugale, S. B. Bari, Eur. J. Med. Chem. 2014, 80, 447-501. DOI:10.1016/j.ejmech.2014.04.072

13. D. Wang, F. Gao, Chem. Centr. J. 2013, 7, 95.

14. A. K. Bilyi, L. M. Antypenko, V. V. Ivchuk, O. M. Kamyshnyi, N. M. Polishchuk, S. I. Kovalenko, ChemPlusChem 2015, 80, 980-989. DOI:10.1002/cplu.201500051

15. L. M. Antypenko, S. I. Kovalenko, A. M. Katsev, E. Z. Komarovska-Porokhnyavets, V. P. Novikov, N. S. Fedyunina, Curr. Comput. Aided Drug Des. 2015, 12, 29-41.

DOI:10.2174/1573409912666160126142236

16. O. M. Antypenko, L. M. Antypenko, S. I. Kovalenko, A. M.
Katsev, O. M. Achkasova, Arab. J. Chem. 2016, 9, 792-805. DOI:10.1016/j.arabjc.2014.09.009

17. Y. A.-M.El-Badry, E. Nassar, M. A.-A.El-Hashash,Eur. J.Chem. 2016, 7, 128-134. DOI:10.5155/eurjchem.7.1.128-134.1370

18. O. M. Antypenko, S. I. Kovalenko, O. V. Karpenko, V. O. Nikitin, L. M. Antypenko, Helv. Chim. Acta 2016, 99, 621-631. DOI:10.1002/hlca.201600062

19. J. C. Burbiel, W. Ghattas, P. Küppers, M. Köse, S. Lacher, A.-M. Herzner, C. E. Müller, ChemMedChem. 2016, 11, 2272-2286. DOI:10.1002/cmdc.201600255

20. M. M. Zeydi, N. Montazeri, M. Fouladi, J. Heterocycl. Chem. 2017, 54, 3549-3553. DOI:10.1002/jhet.2979

21. W. A. Ewes, M. A. Elmorsy, S. M. El-Messery, M. N. A. Nasr, Bioorg. Med. Chem. 2020, 28, 115373.

DOI:10.1016/j.bmc.2020.115373

22. European convention for the protection of vertebrate animal used for experimental and other scientific purposes, Council of Europe, Strasbourg, 1986.

23. J. C. Fehrenbacher, M. R. Vasko, D. B. Duarte, Curr. Protoc. Pharmacol. 2012, 56, 5.4.1-5.4.7. DOI:10.1002/0471141755.ph0506s56

24. S. N. Lapach, A. V.Chubenko, P. N. Babich, Statistical methods in biomedical research using EXCEL, Morion, Ukraine, 2001, 408.

25. E. Breitmaier, Structure elucidation by NMR in organic chemistry: A practical guide, 3rd Ed., Wiley, Germany, 2002, p. 270. DOI:10.1002/0470853069

\section{Povzetek}

V prispevku opisujemo sintezo hidrazidov iz kinazolin-4(3H)-ilidenhidrazinov in dikarboksilnih kislin ter njihove nadaljnje transformacije. Pokazali smo, da tovrstne hidrazide lahko pripravimo s pomočjo aciliranja izhodnega kinazolin-4(3H)-ilidenhidrazina $\mathrm{z}$ ustreznimi acilhalidi, cikličnimi anhidridi in imidazoli monoestrov dikarboksilnih kislin. Pripravljene hidrazide smo pretvorili v $[1,2,4]$ triazolo[1,5-c]kinazoline, ki smo jih uporabili kot izhodne spojine za nadaljnje kemijske modifikacije s ciljem uvedbe amidnega fragmenta v končne molekule. IR in ${ }^{1} \mathrm{H}$ NMR spektroskopija ter sklopljena kromatografsko-masna spektrometrija so omogočile študij strukture produktov. Za pripravljene spojine smo določili tudi protivnetno učinkovitost s pomočjo modela vnetja podganje tačke s karaginanom. Zaključimo lahko, da so ([1,2,4]triazolo[1,5-c]kinazolin-2-il)alkil karboksilne kisline obetavna skupina molekul s protivnetnim delovanje, primerne za nadaljnje poglobljene študije sintez in protivnetnih aktivnosti.

Except when otherwise noted, articles in this journal are published under the terms and conditions of the Creative Commons Attribution 4.0 International License 